\title{
LKB1 in lung cancerigenesis: a serine/threonine kinase as tumor suppressor
}

\author{
Yijun Gao', Gaoxiang $\mathrm{Ge}^{2 \bowtie}$, Hongbin $\mathrm{Ji}^{1 凶}$ \\ ${ }^{1}$ Laboratory of Molecular Cell Biology, Institute of Biochemistry and Cell Biology, Shanghai Institutes for Biological Sciences, \\ Chinese Academy of Sciences, Shanghai 200031, China \\ 2 State Key Laboratory of Molecular Biology, Institute of Biochemistry and Cell Biology, Shanghai Institutes for Biological \\ Sciences, Chinese Academy of Sciences, Shanghai 200031, China \\ $\triangle$ Correspondence: hbji@sibs.ac.cn (H. Ji), gxge@sibs.ac.cn (G. Ge) \\ Received January 28, 2011 Accepted February 12, 2011
}

\begin{abstract}
Lung cancer is featured with high mortality, with a $15 \%$ five-year survival rate worldwide. Genetic alterations, such as loss of function of tumor suppressor genes, frequently contribute to lung cancer initiation, progression and metastasis. Liver kinase B1 (LKB1), as a serine/ threonine kinase and tumor suppressor, is frequently mutated and inactivated in non-small cell lung cancer (NSCLC). Recent studies have provided strong evidences that LKB1 loss promotes lung cancerigenesis process, especially lung cancer progression and metastasis. This review will summarize recent progress on how LKB1 modulates the process of lung cancerigenesis, emphasizing on LKB1 downstream signaling pathways and biological functions. We will further discuss the potential development of prognostic biomarkers or therapeutic targets in lung cancer clinic based on the molecular alteration associated with deregulated LKB1 signaling.
\end{abstract}

\section{KEYWORDS liver kinase B1, lung cancer, tumor suppressor}

\section{INTRODUCTION}

Lung cancer is the leading cause of cancer-related death, with non-small-cell lung cancer (NSCLC) accounting for approximately $85 \%$ of cases. Recent advances in genetic characterization of NSCLC have identified key genes and pathways that are deregulated in these tumors, including gain-of-function mutations of oncogenes, e.g., KRAS and epidermal growth factor receptor (EGFR), as well as loss-offunction mutations of tumor suppressor genes, e.g. tumor protein P53 (TRP53) and liver kinase B1 (LKB1). LKB1, also known as serine-threonine kinase 11 (STK11), belongs to calcium/calmodulin regulated kinase-like family, which is part of the $\mathrm{Ca}^{2+}$ /calmodulin kinase group. Human LKB1 has 433 amino acids while mouse homolog has 436 amino acids. The functional domains of human LKB1 consist of a kinase domain (residues 49-309) and a nuclear localization signal located in the $\mathrm{N}$-terminal non-catalytic region (residues 38-43) (Alessi et al., 2006). LKB1 was identified to be the causative gene responsible for the autosomal dominant Peutz-Jeghers syndrome (PJS), characterized by benign gastrointestinal polyp development and increased risk of malignancy transformation (Hemminki et al., 1998; Jenne et al., 1998; Hemminki, 1999). Although rare in most types of human cancers (Sanchez-Cespedes, 2007; Wingo et al., 2009), LKB1 loss-of-function somatic mutations are frequently observed in NSCLCs $(20 \%-30 \%)$, ranking $L K B 1$ as the third most frequently mutated gene in lung adenocarcinoma after TRP53 and Kras (Sanchez-Cespedes et al., 2002; $\mathrm{Ji}$ et al., 2007; Matsumoto et al., 2007; Weir et al., 2007; Ding et al., 2008). Consistent to its role as a tumor suppressor, in an oncogenic $K$ ras $^{G 12 D}$ driven lung cancer mouse model, concomitant loss of $L K B 1$ significantly accelerated lung cancer progression and metastasis (Ji et al., 2007). More recent studies have established LKB1 as a major player in lung cancer, especially in the metastasis process. Below we will summarize recent progress on the biological functions of LKB1 and further highlight LKB1-dependent molecular pathways that contribute to lung cancer development and progression when compromised.

\section{LKB1 SOMATIC MUTATIONS IN LUNG CANCER}

The LKB1 tumor suppressor gene was first shown to be 
causative for the familial disease Peutz-Jeghers syndrome through linkage analysis (Tomlinson and Houlston, 1997; Jenne et al., 1998). PJS patients harboring germline loss-offunction mutations of $L K B 1$ are more susceptible to malignancy development mainly in gastrointestinal tract during their middle age (Giardiello et al., 1987). Original data showed that somatic mutation of $L K B 1$ is not commonly observed in malignancy outside of gastrointestinal tract (Avizienyte et al., 1999), except for lung cancer. Sanchez-Cespedes et al. firstly reported that $33 \%$ ( 6 of 20 primary tumors and 2 of 4 cell lines) of lung adenocarcinoma had LKB1 somatic mutations (Sanchez-Cespedes et al., 2002). This was confirmed and extended by several other groups showing that the LKB1 inactivating mutation rate ranges from $17 \%$ to $54 \%$ in lung adenocarcinomas (Carretero et al., 2004; Ji et al., 2007; Matsumoto et al., 2007; Koivunen et al., 2008). We and others further showed that $L K B 1$ inactivation mutations prevail in multiple subtypes of NSCLC besides adenocarcinoma ( $\mathrm{Ji}$ et al., 2007; Matsumoto et al., 2007; Koivunen et al., 2008), e.g., LKB1 mutations occur in $19 \%$ of squamous cell carcinomas (8/42), $14 \%$ of large cell carcinomas (1/7), and $25 \%$ of adenosquamous carcinomas (1/4) (Ji et al., 2007). These data suggest that $L K B 1$ loss-of-function mutation is a common event across all different histological subtypes of NSCLC (Table 1).

Despite approximately $20 \%-30 \%$ of human NSCLCs from Caucasian population harbor the loss of function mutations of $L K B 1$, a lower percentage ( $3 \%-7 \%)$ of $L K B 1$ mutations were found in East Asian population including Japanese, Korean and Chinese (Onozato et al., 2007; Koivunen et al., 2008; Gao et al., 2010a; Sun et al., 2010). Only three out of 100 lung tumors harbor LKB1 mutations in Japanese patients (Onozato et al., 2007), while only $5 \%$ of Korean patients were found with somatic LKB1 mutations (Koivunen et al., 2008). Similarly, our recent study has found that $L K B 1$ somatic mutations were approximately $6.9 \%$ in Chinese lung adenocarcinomas (6/86) (Gao et al., 2010a). Future work is warranted to clarify the ethnic difference of $L K B 1$ mutations in lung cancer.

\section{LKB1 SIGNALING PATHWAYS AND BIOLOGICAL FUNCTIONS}

Multiple signal pathways downstream of LKB1, including AMP-activated protein kinase (AMPK)-mammalian target of rapamycin (mTOR), salt-inducible kinase (SIK)-CREB regulated transcription coactivator 1 (CRTC1) and other AMPKrelated subfamily protein kinases mediated pathways, mediate its diverse biological functions. LKB1 interacts with its partners including the pseudokinase Ste20-like adapter protein (STRAD) and the scaffolding protein MO25, which forms a complex and retains LKB1 in the cytosol. Several kinases, including p90 ribosomal S6 kinase (RSK), protein kinase A (PKA), or ataxia-telengiectasia-mutated (ATM) kinase phosphorylate LKB1 and modulate its function. LKB1 could activate downstream serine-threonine kinases through phosphorylation of a conserved threonine located in the " $T$ loop" of their kinase domain (Katajisto et al., 2007). LKB1 has been implicated as a master regulator of multiple biological processes and signaling pathways, including cell growth, cell cycle progression, cell polarity, migration and metabolism. Hereafter we will summarize our current knowledge of the signaling pathways downstream of LKB1, which are apparently essential for its tumor suppressor function in various cancers including lung cancer.

\section{LKB1 in cell metabolism}

LKB1 has been implicated in glucose metabolism through its regulation on AMPK. During metabolic stress such as energy starvation, LKB1 activates AMPK through direct phosphorylation, which in turn phosphorylates tuberous sclerosis complex protein 2 (TSC2), the GTP activating protein (GAP) of Ras homolog enriched in brain (Rheb), resulting in Rheb inactivation and mTOR inhibition. mTOR promotes protein synthesis, cell growth and viability through two major downstream effectors: the p70 S6 kinase (S6K) and the eukaryotic initiation factor $4 \mathrm{E}$ binding protein 1 (4E-BP1) translational regulator (Shackelford and Shaw, 2009). Hypoxia-inducible factor-1 $\alpha$ (HIF-1 $\alpha$ ), one of the downstream effectors of mTOR, functions as a multi-task oncogene by promoting tumor angiogenesis, metastasis, and glycolytic metabolism (Keith and Simon, 2007). mTOR pathway hyperactivation induced by LKB1 deficiency makes cancer cells vulnerable to inhibitors of mTOR treatment. LKB1-mutant cells were shown to be deficient for AMPK activity and more sensitive to mTOR inhibition under conditions of low cellular energy (Carretero et al., 2007). Consistently, LKB1-deficient lung primary tumors had diminished AMPK activity, at least partially responsible for disordered cell energetic checkpoint control in LKB1 deficient lung cancer.

\section{LKB1 in cell polarity and epithelial-mesenchymal transition}

LKB1 was first identified in a genetic screen in Caenorhabditis elegans for mutants affecting the cytoplasmic partitioning, in which the LKB1 ortholog abnormal embryonic PARtitioning of cytoplasm (par-4) is an essential regulator in asymmetric division during early embryonic development (Kemphues et al., 1988). The role of LKB1 in the establishment of polarity is conserved in different organisms and in different cell types. Translocation of LKB1 from the nucleus to the cytoplasm and the kinase activity of LKB1 are required in establishing cell polarity (Baas et al., 2004; Forcet et al., 2005). LKB1 regulates cell polarity through the AMPK family kinases. Downstream of LKB1, modulation of actin and microtubule cytoskeleton is critical in establishment and 
Table 1 LKB1 mutations in human primary lung tumors and cancer cell lines

\begin{tabular}{|c|c|c|c|c|c|}
\hline Sample type & Histology & Mutations & Mutation rate & Mutation type & References \\
\hline \multirow[t]{18}{*}{ Tumors } & $A D$ & $8 \%$ & $1 / 12$ & Mutation & Avizienyte et al., 1999 \\
\hline & & $30 \%$ & $6 / 20$ & Mutation & Sanchez-Cespedes et al., 2002 \\
\hline & & $4.50 \%$ & $7 / 155$ & Mutation/Deletion & Matsumoto et al., 2007 \\
\hline & & $4 \%$ & $3 / 81$ & Mutation/Deletion & Onozato et al., 2007 \\
\hline & & $34 \%$ & $27 / 80$ & Mutation/Deletion & Ji et al., 2007 \\
\hline & & $13 \%$ & $13 / 207$ & Mutation/Deletion & Koivunen et al., 2008 \\
\hline & & $6.90 \%$ & $6 / 86$ & Mutation/Deletion & Gao et al., 2010a \\
\hline & & 0 & $0 / 52$ & & Sun et al., 2010 \\
\hline & SCC & 0 & $0 / 12$ & & Avizienyte et al., 1999 \\
\hline & & 0 & $0 / 12$ & & Sanchez-Cespedes et al., 2002 \\
\hline & & 0 & $0 / 14$ & & Onozato et al., 2007 \\
\hline & & $19 \%$ & $8 / 42$ & Mutation/Deletion & Ji et al., 2007 \\
\hline & & $5 \%$ & $5 / 92$ & Mutation/Deletion & Koivunen et al., 2008 \\
\hline & LCC & 0 & $0 / 3$ & & Avizienyte et al., 1999 \\
\hline & & 0 & $0 / 2$ & & Onozato et al., 2007 \\
\hline & & $14 \%$ & $1 / 7$ & Mutation & Ji et al., 2007 \\
\hline & SCLC & 0 & $0 / 1$ & & Avizienyte et al., 1999 \\
\hline & & 0 & $0 / 1$ & & Onozato et al., 2007 \\
\hline \multirow[t]{12}{*}{ Cell lines } & $A D$ & $50 \%$ & $2 / 4$ & Mutation & Sanchez-Cespedes et al., 2002 \\
\hline & & $54 \%$ & $6 / 11$ & Mutation/Deletion & Carretero et al., 2004 \\
\hline & & $42 \%$ & $13 / 31$ & Mutation/Deletion & Matsumoto et al., 2007 \\
\hline & & $38 \%$ & $3 / 8$ & Mutation/Deletion & Onozato et al., 2007 \\
\hline & SCC & 0 & $0 / 2$ & & Carretero et al., 2004 \\
\hline & & $27 \%$ & $3 / 11$ & Deletion & Matsumoto et al., 2007 \\
\hline & & 0 & $0 / 6$ & & Onozato et al., 2007 \\
\hline & LCC & $43 \%$ & $3 / 7$ & Mutation/Deletion & Matsumoto et al., 2007 \\
\hline & & $33 \%$ & $1 / 3$ & Mutation & Onozato et al., 2007 \\
\hline & SCLC & 0 & $0 / 11$ & & Carretero et al., 2004 \\
\hline & & $5 \%$ & $1 / 19$ & Deletion & Matsumoto et al., 2007 \\
\hline & & 0 & $0 / 5$ & & Onozato et al., 2007 \\
\hline
\end{tabular}

AD: adenocarcinoma; SCC: squamous cell carcinoma; LCC: large cell carcinoma; SCLC: small cell lung cancer.

maintenance of cell polarity in several cell types. This requires AMPK-mediated phosphorylation of myosin regulatory light chain (MRLC) (Lee et al., 2007), as well as the AMPK-related kinase microtubule affinity-regulating kinases (MARKs)mediated phosphorylation of microtubule-associated proteins (Hezel et al., 2008). More recently, a novel LKB1-Cdc42-p21activated kinase (PAK) pathway has been proposed to regulate cell polarity in NSCLC (Zhang et al., 2008). LKB1 colocalizes with cell division cycle 42 (cdc42) and p21 protein (Cdc42/Rac)-activated kinase (PAK) at the cellular leading edge upon stimulation, which leads to increased PAK phosphorylation and the promotion of downstream cell polarity events. LKB1 loss reduces PAK1 and Cdc42 activity, resulting in aberrant cell polarity. In human lung cancer cells LKB1 loss induces epithelial-mesenchymal transition (EMT) through zinc finger E-box binding homeobox 1 (ZEB1), which transcriptionally represses E-cadherin expression (Roy et al., 2010). Moreover, LKB1 inhibits EMT through its negative regulatory effects on transforming growth factor- $\beta$ (TGF- $\beta$ ) signaling (Moren et al., 2011). LKB1 directly binds to scaffolding protein LKB1-interacting protein 1 (LIP1), which recruits co-Smad Smad4, the essential TGF- $\beta$ signaling component. By forming an LKB1-LIP-Smad4 complex, LKB1 prevents Smad4-dependent transcription and subsequent TGF- $\beta$ signal transduction. Cell polarity is critical in maintenance of the epithelium integrity and function. Loss of 
cell polarity is one of the hallmarks of the EMT process and correlates with invasive behavior of malignant cells in human cancers (Wu and Zhou, 2008). In concert with this notion, compromised LKB1 signaling could trigger aberrant polarity and EMT induction, which is a prerequisite for enhanced migration and invasive behavior of cancer cells.

\section{LKB1 in cell cycle regulation}

LKB1 induces cell growth arrest at G1 phase mainly through TRP53 and its target gene cyclin-dependent kinase inhibitor $1 \mathrm{~A}\left(\mathrm{p} 21^{\mathrm{WAF} 1 / \mathrm{CIP} 1}\right.$ ) (Jimenez et al., 2003). Ectopic expression of LKB1 in A549 leads to $G 1$ to $S$ cell cycle arrest. Mechanistic study further pointed out that LKB1 functions through the activation of AMPK to phosphorylate TRP53 at Ser15 and induce the expression of downstream cyclin-dependent kinase inhibitors $\mathrm{p} 21^{\mathrm{WAF} 1 / \mathrm{CIP} 1}$ and cyclin-dependent kinase inhibitor 1B (p27) (Jones et al., 2005). Further studies demonstrate that LKB1 itself binds to and stabilizes TRP53 in the nucleus, resulting in TRP53-dependent cell cycle G1 arrest (Zeng and Berger, 2006). In addition, LKB1 has been shown to bind to and regulate brahma-related gene 1 (Brg1) (Marignani et al., 2001), which is involved in retinoblastoma protein (RB) induced cell cycle arrest in both $\mathrm{G} 1$ and $\mathrm{S}$ phases, suggesting an alternative mechanism for LKB1 in inducing cell growth arrest. Loss of heterozygosity ( $\mathrm{LOH})$ of the chromosome 19p region containing both LKB1 and BRG1 were frequently observed in lung cancers (Rodriguez-Nieto and Sanchez-Cespedes, 2009), indicating a non-overlapping function of these two tumor suppressor genes.

\section{LKB1 in cell apoptosis}

The role of LKB1 in cell apoptosis has been well established in past decades. Karuman et al. firstly demonstrated that LKB1 could induce apoptosis in epithelial cells via its kinase activity. Further work reveals that LKB1 functions through physical interaction with TRP53. Consistently, the polyps from PJS patients with LKB1 mutation showed reduced rate of cell apoptosis (Karuman et al., 2001). Gene expression profiling of A549 lung cancer cells with ectopic LKB1 expression reveals deregulation of many genes involved in cell proliferation and apoptosis, in which the role of TRP53 is also highlighted (Jimenez et al., 2003). Interestingly, studies suggest LKB1 functions through AMPK to protect cells from apoptosis under energy stress (Shaw et al., 2004; Inge et al., 2009). Under energy stress induced by glucose withdrawal or addition of glucose analog-2-deoxyglucose, LKB1-deficient cells are unable to shut down energy consuming process, thus becoming hypersensitive to apoptosis induced by drugs that increase cellular AMP/ATP ratio. The discrepancy concerning the role of LKB1 in cell apoptosis may be dependent on the metabolic status and the cell types.

\section{LKB1 in autophagy}

LKB1 regulates autophagy mainly through mTOR, which controls the expression of autophagy-related molecules at both transcriptional and translational levels. The activation of mTOR under nutrient-rich conditions suppresses autophagy and promotes cell survival and proliferation. In response to energy stress, LKB1 activates AMPK to repress mTOR signaling, which thereafter triggers autophagic process. Recent study has validated a promotive role of LKB1-AMPK pathway in autophagy through phosphorylation and stabilization of cyclin-dependent kinase inhibitor P27. Under stress conditions, the LKB1-AMPK pathway is activated and stabilizes p27 through phosphorylation at Thr198 and promotes cell survival through entering autophagy (Liang et al., 2007). Moreover, LKB1 is also involved in hydrogen peroxide $\left(\mathrm{H}_{2} \mathrm{O}_{2}\right.$ )-induced autophagy promoted by Poly(ADPribose) polymerase-1 (PARP-1) activation (Huang et al., 2009). More recently, LKB1-AMPK pathway was reported to be activated by ataxia telangiectasia mutated (ATM) in response to elevated reactive oxygen species (ROS), leading to tuberous sclerosis 2 (TSC2) activation and mTOR repression and induction of autophagy (Alexander et al., 2010). Thus, under metabolic stress, LKB1 activation protects the cells from death through autophagy.

\section{LKB1 IN LUNG CANCERIGENESIS}

As a multi-functional kinase and tumor suppressor, LKB1 is involved in a broad spectrum of cellular activity. Despite of the well documented high frequency of LKB1 loss-of-function mutations in lung cancer in the past decade, we have not gained much knowledge about the role of LKB1 in lung cancerigenesis until very recently from the work in lung cancer mouse model. We will summarize and discuss the roles of LKB1 in lung cancer initiation, trans-differentiation and metastasis.

\section{LKB1 in lung tumor initiation}

Early studies have suggested the role of LKB1 in tumor initiation in many epithelial malignancies, including gastric hamartomas, hepatocellular carcinomas, breast cancer, endometrial tumors and pancreatic neoplasm. Miyoshi et al. first reported the heterozygous deletion of $L k b 1$ in mice is sufficient to the development of gastrointestinal polyps after $>20$ weeks of age (Miyoshi et al., 2002). Another evidence came from the observation of hepatocellular carcinoma (HCC) in $L k b 1$ heterozygous mice, which is mainly caused by $L k b 1 \mathrm{LOH}$ (Nakau et al., 2002). Lkb1 deletion in the mammary gland also induces breast tumor formation with a latency of 46-85 weeks (McCarthy et al., 2009). Similarly, mice with $L k b 1$ deletion specifically in the pancreatic epithelium developed pancreatic serous cystadenomas 
(Hezel et al., 2008). Lkb1 inactivation results in malignant transformation of endometrium and is sufficient to promote the development of invasive endometrial cancer (Contreras et al., 2008). The entire endometrium underwent extrauterine spread after $L k b 1$ loss, suggesting an essential role of $L k b 1$ in driving tumor initiation and progression. However, deletion of $L k b 1$ alone in mouse lungs for more than 50 weeks does not lead to tumor formation (Ji et al., 2007). This discrepancy may reflect the context of different signaling crosstalk with $L k b 1$ signaling in a tissue-specific manner.

\section{LKB1 in lung tumor trans-differentiation}

Kras is a key oncogene frequently mutated in NSCLC. Active KRAS expression in mouse lungs specifically drives the formation of only one specific pathological subtype of lung cancer, lung adenocarcinoma (Jackson et al., 2001). Interestingly, we found that the concomitant loss of $L k b 1$ confers lung adenocarcinoma the ability to trans-differentiate into squamous cell carcinoma (Ji et al., 2007). Mutation of $L K B 1$ in human lung squamous cell carcinoma occurs at a frequency similar to that in lung adenocarcinoma ( 20\%) (Ji et al., 2007). Although the mechanism remains elusive, we reason that LKB1 deficiency may confer the lung cancer cell with stemness allowing the trans-differentiation from adenocarcinoma to squamous cell carcinoma. Despite that the role of LKB1 in lung stem cell has not been reported, this notion is indirectly supported by recent studies that highlighted the essential role of LKB1 in hemapoietic stem cell (HSC) homeostasis and survival (Gan et al., 2010; Gurumurthy et al., 2010; Nakada et al., 2010). We, however, cannot exclude the possibility that LKB1 loss could potentiate the survival and transformation of basal cells, from which the squamous cell carcinoma derives.

\section{LKB1 in lung cancer metastasis}

Two human genetic analyses have indicated the potential roles of LKB1 in metastasis. Sobottka and colleagues found $\mathrm{LOH}$ at the $L K B 1$ locus in $58 \%$ of metastatic lung tumors, in which no somatic mutation has been detected (Sobottka et al., 2000). A second study showed that $L K B 1$ mutation was seen in only $1 \%$ of stage I NSCLC but in $12 \%$ of brain metastasis (Matsumoto et al., 2007). Taking advantage of animal models, we have demonstrated that LKB1 loss in mice dramatically promoted lung cancer metastasis ( $\mathrm{Ji}$ et al., 2007), similar to that from TRP53 loss. We found that homozygous $L k b 1$ deletion increases the penetrance of lymph node and distant metastases in this mouse model ( $\mathrm{Ji}$ et al., 2007). Gene expression profiling in human lung cancer cell lines and mouse lung tumors with or without LKB1 identified a variety of metastasis-promoting genes, including neuronal precursor cell-expressed, developmentally downregulated gene 9 (NEDD9) as downstream target of LKB1.
NEDD9 is a scaffold protein without catalytic activity. High expression of NEDD9 has been reported in human melanoma and breast cancer (O'Neill et al., 2007). Overexpression of NEDD9 promotes pulmonary metastasis of melanoma cells. Consistently, NEDD9 RNAi in A549 cells significantly reduced cell migration and invasion ( $\mathrm{Ji}$ et al., 2007). These findings suggest that NEDD9 is potentially an important mediator of metastasis downstream of LKB1. In addition to the cancercell-autonomous mechanisms, our recent study has highlighted an essential role of tumor stroma remodeling in LKB1 deficiency-mediated lung cancer metastasis. Cancer progression is a multi-step process involving interplay between tumor cells and surrounding stroma, including extracellular matrix (ECM). We have identified lysyl oxidase (LOX) as an essential mediator of LKB1-deficiency-elicited lung cancer progression through ECM alteration, especially collagen matrix remodeling (Gao et al., 2010b). As a key enzyme in collagen cross-linking, increased LOX activity in LKB1deficient lung tumors results in excess collagen deposition, which promotes cancer cell invasion through activation of v-src sarcoma (Schmidt-Ruppin A-2) viral oncogene homolog (SRC) and focal adhesion kinase (FAK) downstream of $\beta 1$ integrin signaling. This is further proven by integrative genomic and proteomic analyses that SRC and FAK signaling pathway activation were associated with $L k b 1$ loss and with progression to invasive and metastatic lung tumors (Carretero et al., 2010). LKB1 loss triggered the up-regulation of LOX expression through the mTOR-HIF-1 $\alpha$ signaling axis. Vascular endothelial growth factor (VEGF), another downstream target regulated by HIF-1 $\alpha$, is well known to promote tumor angiogenesis. Cyclooxygenase-2 (COX2) is another important target downstream of LKB1 and its expression is significantly increased in polyps from $L k b 1^{+/-}$mice. LKB1 inhibits COX2 transcription through phosphorylation and degradation of its upstream transcriptional activator polyomavirus enhancer activator-3 (PEA3) (Upadhyay et al., 2006). Cyclooxygenase-2-mediated production of prostaglandin $\mathrm{E}_{2}\left(\mathrm{PGE}_{2}\right)$ provided a pro-survival microenvironmental cue for the tumor cells by inhibiting apoptosis, suppression of antitumor immunity and promoting angiogenesis, which eventually lead to cancer progression and metastasis. Taken together, LKB1 deficiency promotes lung cancer metastasis, via both cancer-cell autonomous and non-cancer-cell autonomous mechanisms.

\section{POTENTIAL TARGETS FOR CANCER THERAPY}

The observation of high mutation frequency of $L K B 1$ in NSCLCs warrants studies to explore LKB1 downstream molecules as targets for development of effective cancer therapy. LKB1 itself does not represent an ideal drug target since the inhibition of LKB1 promotes cancerigenesis. AMPK mediates signal transduction from LKB1 to mTOR-HIF1a. Therefore, compound activating AMPK, such as metformin, 
phenformin and phosphatidylinositol ether lipid analogues (PIA), might be desirable for cancer treatment (Fig. 1) (Memmott et al., 2008; Shackelford and Shaw, 2009). Since mTOR pathway downstream of LKB1-AMPK is commonly hyperactive in LKB1 deficient tumors even under conditions of low cellular energy, mTOR inhibitors might be of significant therapeutic advantage in lung cancer (Fig. 1). Inhibitors of mTOR have been investigated in lung cancer, showing clear evidences of anticancer activity as single reagent or in combination with chemotherapy and radiation (Mita et al., 2008). Combination of specific therapies targeting parallel pathways might improve effectiveness as LKB1 mutation frequently accompanies with KRAS activation in lung cancer. Based on this scenario, Lkb1/Kras mutant lung cancers are with increased sensitivity to combined therapy simultaneously targeting mTOR and MAP kinase kinase (MEK), effectors downstream of RAS (Mahoney et al., 2009). Recently, combinational inhibition of SRC, phosphoinositide 3-kinases (PI3K) and MEK1/2 also showed synergistic effect in tumor regression of $L k b 1$-deficient primary and metastatic lung tumors (Carretero et al., 2010). These findings highlight the prospective of applying unique combinatorial therapies for lung cancer treatment. Previous studies have suggested LKB1 loss could promote tumor angiogenesis through induction of VEGF downstream of mTOR-HIF1a (Fig. 1). Anti-angiogenic drugs may have potential benefit in disease treatment. Sunitinib inhibition of VEGFR kinase activity resulted in a prolonged survival in $K r a s, L k b 1^{L / L}$ lung cancer mice mainly through suppression of primary tumor growth without much influence on the malignancy progression (Gandhi et al., 2009). Our recent work identified LOX as another downstream target of LKB1-mTOR-HIF1 $\alpha$ pathway (Gao et al., 2010b). LOX activity inhibition by pharmacological inhibitor $\beta$-aminopropionitrile (BAPN) significantly alleviates LKB1-deficient lung cancer malignancy and invasion, indicating LOX is a potentially important therapeutic target for lung cancer treatment (Fig. 1). We further show LOX serum activity is elevated in metastatic lung cancers, providing evidence that LOX can be a promising biomarker for lung cancer prognosis. As an important mediator downstream of LKB1, elevated COX-2 has been reported in many malignancies including lung cancer. In addition, increased COX-2 is associated with decreased survival in lung cancer patients (Achiwa et al., 1999). Induction of COX-2 has been implicated in promotion of tumor formation and progression. COX2 target therapy has been implicated in preclinical animal models. COX2 specific inhibitors (celecoxib, rofecoxib) are reported to have anti-tumor activity and clinical efficacy of COX-2 inhibitors in the treatment of NSCLC is presently under evaluation in combination with chemotherapy and/or radiotherapy (Fig. 1) (Spano et al., 2004). LKB1 loss also altered cellular metabolism in cancer cells. Cells that lack LKB1 fail to respond to energetic stress and undergo cell death, providing therapeutic chance for drugs inhibiting cellular metabolism.

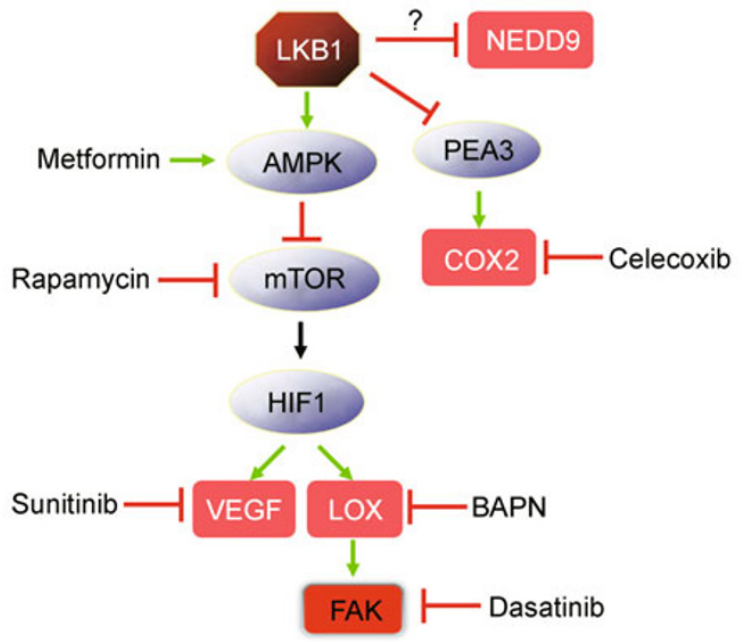

Figure 1. Schematic illustration of LKB1 signaling network and potential downstream targets for cancer therapy. LKB1 inhibits lung cancer progression and metastasis potentially through signaling pathways including: (1) The classical AMPK-mTOR-HIF1a pathway, of which LOX and VEGF are two main downstream targets. AMPK agonist and small molecule inhibitors targeting mTOR, LOX and VEGFR are shown. (2) LKB1-PEA3-COX2 pathway. COX2 inhibition by celecoxib has been implicated in NSCLC clinical treatment. (3) NEDD9 is an important mediator of metastasis downstream of LKB1 with unknown mechanism.

LKB1-null NSCLC cell lines were more sensitive to 2deoxyglucose treatment compared to those with wild-type LKB1 (Inge et al., 2009), indicating glucose analog, which induces energetic stress may benefit those lung cancer patients with LKB1 deficiency. As discussed above, epigenetic control may be also involved in silencing LKB1 in lung cancer. Efforts into upstream signaling or regulation of LKB1 expression will definitely provide promising insights for the development of novel therapeutic strategies in clinic.

\section{PROSPECTIVES}

In summary, we have gained tremendous knowledge about the essential roles of LKB1 in lung cancerigenesis at both signaling pathway and cancer biology standpoints in last decades. Nonetheless, there are still many important questions remained to be answered. Firstly, a major challenge for future research is to identify those essential substrates and interacting partners of LKB1 in lung cancer. Further functional and mechanistic characterization of these substrates of LKB1 would definitely provide us important insights into the fundamental mechanisms by which LKB1 regulates cell proliferation, metabolism, polarity and cancerigenesis. This will no doubt give rise to the development of new approaches for anticancer treatments. Second, it is interesting to dissect 
the association of $\angle K B 1$ mutation and other genetic alteration in human lung cancer. $L K B 1$ loss is frequently accompanied with KRAS activation mutations in NSCLC. Loss-of-function of $L K b 1$ synergizes with $K$ ras mutation in mouse lung cancer progression and metastasis. Interestingly, LKB1 mutation is mutually exclusive with EGFR mutations in NSCLC (Ding et al., 2008). Whether this is due to the signaling abundance of these two genetic alterations or it is a simple reflection of the difference in their association with smoking status has not been determined. Last, the ethnical difference of $L K B 1$ mutation rate between Caucasian and East Asian populations warrants further detailed genetic analysis. Is it possible that the epigenetic control or allelic loss of LKB1 explains this? Answers to all these key questions will not only improve our current understanding of LKB1 as an essential tumor suppressor in lung cancer but provide potential targets for clinical therapeutic strategy development.

\section{ACKNOWLEDGEMENTS}

The authors thank Drs. Tian Xiao and Fuming Li and Ms. Wenjing Zhang for helpful discussion and critical proofreading. This work was supported by the National Basic Research Program of China (Grant No. 2010CB912102), the National Natural Science Foundation of China (Grant Nos. 30871284, 30971461 and 30971495), and the Science and Technology Commission of Shanghai Municipality (No. 09JC1416300). H.J. and G.G. are scholars of the Hundred Talents Program of the Chinese Academy of Sciences.

The authors declare no financial conflict of interests.

\section{ABBREVIATIONS}

AMPK, AMP-activated protein kinase; ATM, ataxia-telengiectasiamutated; COX2; cyclooxygenase-2; ECM, extracellular matrix; EGFR, epidermal growth factor receptor; EMT, epithelial-mesenchymal transition; FAK, focal adhesion kinase; HIF-1 $\alpha$, hypoxia-inducible factor-1 $\alpha$; HSC, hemapoietic stem cell; LIP1, LKB1interacting protein 1; LKB1, liver kinase B1; LOH, Loss of heterozygosity; LOX, lysyl oxidase; mTOR, mammalian target of rapamycin; NSCLC, non-small cell lung cancer; PEA3, polyomavirus enhancer activator-3; $\mathrm{PGE}_{2}$, prostaglandin $\mathrm{E}_{2}$; PIA, phosphatidylinositol ether lipid analogues; PI3K, phosphoinositide 3-kinases; PJS, PeutzJeghers syndrome; PKA, protein kinase $A ;$ ROS, reactive oxygen species; STK11, serine-threonine kinase 11; TGF- $\beta$, transforming growth factor- $\beta$; TRP53, tumor protein P53; TSC2, tuberous sclerosis 2; VEGF, vascular endothelial growth factor; ZEB1, zinc finger E-box binding homeobox 1

\section{REFERENCES}

Achiwa, H., Yatabe, Y., Hida, T., Kuroishi, T., Kozaki, K., Nakamura, S., Ogawa, M., Sugiura, T., Mitsudomi, T., and Takahashi, T. (1999). Prognostic significance of elevated cyclooxygenase 2 expression in primary, resected lung adenocarcinomas. Clin Cancer Res 5, 1001-1005.

Alessi, D.R., Sakamoto, K., and Bayascas, J.R. (2006). LKB1dependent signaling pathways. Annu Rev Biochem 75, 137-163.
Alexander, A., Cai, S.L., Kim, J., Nanez, A., Sahin, M., MacLean, K. H., Inoki, K., Guan, K.L., Shen, J., Person, M.D., et al. (2010). ATM signals to TSC2 in the cytoplasm to regulate mTORC1 in response to ROS. Proc Natl Acad Sci U S A 107, 4153-4158.

Avizienyte, E., Loukola, A., Roth, S., Hemminki, A., Tarkkanen, M., Salovaara, R., Arola, J., Bützow, R., Husgafvel-Pursiainen, K., Kokkola, A., et al. (1999). LKB1 somatic mutations in sporadic tumors. Am J Pathol 154, 677-681.

Baas, A.F., Kuipers, J., van der Wel, N.N., Batlle, E., Koerten, H.K., Peters, P.J., and Clevers, H.C. (2004). Complete polarization of single intestinal epithelial cells upon activation of LKB1 by STRAD. Cell 116, 457-466.

Carretero, J., Medina, P.P., Blanco, R., Smit, L., Tang, M., Roncador, G., Maestre, L., Conde, E., Lopez-Rios, F., Clevers, H.C., et al. (2007). Dysfunctional AMPK activity, signalling through mTOR and survival in response to energetic stress in LKB1-deficient lung cancer. Oncogene 26, 1616-1625.

Carretero, J., Medina, P.P., Pio, R., Montuenga, L.M., and SanchezCespedes, M. (2004). Novel and natural knockout lung cancer cell lines for the LKB1/STK11 tumor suppressor gene. Oncogene 23, 4037-4040.

Carretero, J., Shimamura, T., Rikova, K., Jackson, A.L., Wilkerson, M.D., Borgman, C.L., Buttarazzi, M.S., Sanofsky, B.A., McNamara, K.L., Brandstetter, K.A., et al. (2010). Integrative genomic and proteomic analyses identify targets for Lkb1-deficient metastatic lung tumors. Cancer Cell 17, 547-559.

Contreras, C.M., Akbay, E.A., Gallardo, T.D., Haynie, J.M., Sharma, S., Tagao, O., Bardeesy, N., Takahashi, M., Settleman, J., Wong, K.K., et al. (2008). Lkb1 inactivation is sufficient to drive endometrial cancers that are aggressive yet highly responsive to mTOR inhibitor monotherapy. Dis Models \& Mechan 3, 181-193.

Contreras, C.M., Gurumurthy, S., Haynie, J.M., Shirley, L.J., Akbay, E.A., Wingo, S.N., Schorge, J.O., Broaddus, R.R., Wong, K.K., Bardeesy, N., et al. (2008). Loss of Lkb1 provokes highly invasive endometrial adenocarcinomas. Cancer Res 68, 759-766.

Ding, L., Getz, G., Wheeler, D.A., Mardis, E.R., McLellan, M.D., Cibulskis, K., Sougnez, C., Greulich, H., Muzny, D.M., Morgan, M. B., et al. (2008). Somatic mutations affect key pathways in lung adenocarcinoma. Nature 455, 1069-1075.

Forcet, C., Etienne-Manneville, S., Gaude, H., Fournier, L., Debilly, S., Salmi, M., Baas, A., Olschwang, S., Clevers, H., and Billaud, M. (2005). Functional analysis of Peutz-Jeghers mutations reveals that the LKB1 C-terminal region exerts a crucial role in regulating both the AMPK pathway and the cell polarity. Hum Mol Genet 14, 1283-1292.

Gan, B., Hu, J., Jiang, S., Liu, Y., Sahin, E., Zhuang, L., FletcherSananikone, E., Colla, S., Wang, Y.A., Chin, L., et al. (2010). Lkb1 regulates quiescence and metabolic homeostasis of haematopoietic stem cells. Nature 468, 701-704.

Gandhi, L., McNamara, K.L., Li, D., Borgman, C.L., McDermott, U., Brandstetter, K.A., Padera, R.F., Chirieac, L.R., Settleman, J.E., and Wong, K.K. (2009). Sunitinib prolongs survival in genetically engineered mouse models of multistep lung carcinogenesis. Cancer Pre Res (Phila), Pa 2, 330-337.

Gao, B., Sun, Y., Zhang, J., Ren, Y., Fang, R., Han, X., Shen, L., Liu, X.Y., Pao, W., Chen, H., et al. (2010a). Spectrum of LKB1, EGFR, and KRAS mutations in chinese lung adenocarcinomas. J Thorac Oncol 5, 1130-1135. 
Gao, Y., Xiao, Q., Ma, H., Li, L., Liu, J., Feng, Y., Fang, Z., Wu, J., Han, X., Zhang, J., et al. (2010b). LKB1 inhibits lung cancer progression through lysyl oxidase and extracellular matrix remodeling. Proc Natl Acad Sci U S A 107, 18892-18897.

Giardiello, F.M., Welsh, S.B., Hamilton, S.R., Offerhaus, G.J., Gittelsohn, A.M., Booker, S.V., Krush, A.J., Yardley, J.H., and Luk, G.D. (1987). Increased risk of cancer in the Peutz-Jeghers syndrome. N Engl J Med 316, 1511-1514.

Gurumurthy, S., Xie, S.Z., Alagesan, B., Kim, J., Yusuf, R.Z., Saez, B., Tzatsos, A., Ozsolak, F., Milos, P., Ferrari, F., et al. (2010). The Lkb1 metabolic sensor maintains haematopoietic stem cell survival. Nature 468, 659-663.

Hemminki, A. (1999). The molecular basis and clinical aspects of Peutz-Jeghers syndrome. Cell Mol Life Sci 55, 735-750.

Hemminki, A., Markie, D., Tomlinson, I., Avizienyte, E., Roth, S., Loukola, A., Bignell, G., Warren, W., Aminoff, M., Höglund, P., et al. (1998). A serine/threonine kinase gene defective in PeutzJeghers syndrome. Nature 391, 184-187.

Hezel, A.F., Gurumurthy, S., Granot, Z., Swisa, A., Chu, G.C., Bailey, G., Dor, Y., Bardeesy, N., and Depinho, R.A. (2008). Pancreatic LKB1 deletion leads to acinar polarity defects and cystic neoplasms. Mol Cell Biol 28, 2414-2425.

Huang, Q., Wu, Y.T., Tan, H.L., Ong, C.N., and Shen, H.M. (2009). A novel function of poly(ADP-ribose) polymerase-1 in modulation of autophagy and necrosis under oxidative stress. Cell Death Differ 16, 264-277.

Inge, L.J., Coon, K.D., Smith, M.A., and Bremner, R.M. (2009). Expression of LKB1 tumor suppressor in non-small cell lung cancer determines sensitivity to 2-deoxyglucose. J Thorac Cardiovasc Surg 137, 580-586.

Jackson, E.L., Willis, N., Mercer, K., Bronson, R.T., Crowley, D., Montoya, R., Jacks, T., and Tuveson, D.A. (2001). Analysis of lung tumor initiation and progression using conditional expression of oncogenic K-ras. Genes Dev 15, 3243-3248.

Jenne, D.E., Reimann, H., Nezu, J., Friedel, W., Loff, S., Jeschke, R., Müller, O., Back, W., and Zimmer, M. (1998). Peutz-Jeghers syndrome is caused by mutations in a novel serine threonine kinase. Nat Genet 18, 38-43.

Ji, H., Ramsey, M.R., Hayes, D.N., Fan, C., McNamara, K., Kozlowski, P., Torrice, C., Wu, M.C., Shimamura, T., Perera, S.A., et al. (2007). LKB1 modulates lung cancer differentiation and metastasis. Nature 448, 807-810.

Jimenez, A.I., Fernandez, P., Dominguez, O., Dopazo, A., and Sanchez-Cespedes, M. (2003). Growth and molecular profile of lung cancer cells expressing ectopic LKB1: down-regulation of the phosphatidylinositol 3'-phosphate kinase/PTEN pathway. Cancer Res 63, 1382-1388.

Jones, R.G., Plas, D.R., Kubek, S., Buzzai, M., Mu, J., Xu, Y., Birnbaum, M.J., and Thompson, C.B. (2005). AMP-activated protein kinase induces a p53-dependent metabolic checkpoint. Mol Cell 18, 283-293.

Karuman, P., Gozani, O., Odze, R.D., Zhou, X.C., Zhu, H., Shaw, R., Brien, T.P., Bozzuto, C.D., Ooi, D., Cantley, L.C., et al. (2001). The Peutz-Jegher gene product LKB1 is a mediator of p53-dependent cell death. Mol Cell 7, 1307-1319.

Katajisto, P., Vallenius, T., Vaahtomeri, K., Ekman, N., Udd, L., Tiainen, M., and Mäkelä, T.P. (2007). The LKB1 tumor suppressor kinase in human disease. Biochim Biophys Acta 1775, 63-75.
Keith, B., and Simon, M.C. (2007). Hypoxia-inducible factors, stem cells, and cancer. Cell 129, 465-472.

Kemphues, K.J., Priess, J.R., Morton, D.G., and Cheng, N.S. (1988). Identification of genes required for cytoplasmic localization in early C. elegans embryos. Cell 52, 311-320.

Koivunen, J.P., Kim, J., Lee, J., Rogers, A.M., Park, J.O., Zhao, X., Naoki, K., Okamoto, I., Nakagawa, K., Yeap, B.Y., et al. (2008). Mutations in the LKB1 tumour suppressor are frequently detected in tumours from Caucasian but not Asian lung cancer patients. $\mathrm{Br} \mathrm{J}$ Cancer 99, 245-252.

Lee, J.H., Koh, H., Kim, M., Kim, Y., Lee, S.Y., Karess, R.E., Lee, S. H., Shong, M., Kim, J.M., Kim, J., et al. (2007). Energy-dependent regulation of cell structure by AMP-activated protein kinase. Nature 447, 1017-1020.

Liang, J., Shao, S.H., Xu, Z.X., Hennessy, B., Ding, Z., Larrea, M., Kondo, S., Dumont, D.J., Gutterman, J.U., Walker, C.L., et al. (2007). The energy sensing LKB1-AMPK pathway regulates p27 (kip1) phosphorylation mediating the decision to enter autophagy or apoptosis. Nat Cell Biol 9, 218-224.

Mahoney, C.L., Choudhury, B., Davies, H., Edkins, S., Greenman, C., Haaften, G., Mironenko, T., Santarius, T., Stevens, C., Stratton, M.R., et al. (2009). LKB1/KRAS mutant lung cancers constitute a genetic subset of NSCLC with increased sensitivity to MAPK and mTOR signalling inhibition. Br J Cancer 100, 370-375.

Marignani, P.A., Kanai, F., and Carpenter, C.L. (2001). LKB1 associates with $\mathrm{Brg} 1$ and is necessary for $\mathrm{Brg} 1$-induced growth arrest. J Biol Chem 276, 32415-32418.

Matsumoto, S., Iwakawa, R., Takahashi, K., Kohno, T., Nakanishi, Y., Matsuno, Y., Suzuki, K., Nakamoto, M., Shimizu, E., Minna, J.D., et al. (2007). Prevalence and specificity of LKB1 genetic alterations in lung cancers. Oncogene 26, 5911-5918.

McCarthy, A., Lord, C.J., Savage, K., Grigoriadis, A., Smith, D.P., Weigelt, B., Reis-Filho, J.S., and Ashworth, A. (2009). Conditional deletion of the Lkb1 gene in the mouse mammary gland induces tumour formation. J Pathol 219, 306-316.

Memmott, R.M., Gills, J.J., Hollingshead, M., Powers, M.C., Chen, Z., Kemp, B., Kozikowski, A., and Dennis, P.A. (2008). Phosphatidylinositol ether lipid analogues induce AMP-activated protein kinase-dependent death in LKB1-mutant non small cell lung cancer cells. Cancer Res 68, 580-588.

Mita, M., Sankhala, K., Abdel-Karim, I., Mita, A., and Giles, F. (2008). Deforolimus (AP23573) a novel mTOR inhibitor in clinical development. Expert Opin Investig Drugs 17, 1947-1954.

Miyoshi, H., Nakau, M., Ishikawa, T.O., Seldin, M.F., Oshima, M., and Taketo, M.M. (2002). Gastrointestinal hamartomatous polyposis in Lkb1 heterozygous knockout mice. Cancer Res 62, 2261-2266.

Moren, A., Raja, E., Heldin, C.H., and Moustakas, A. (2011). Negative regulation of TGF $\{$ beta\} signaling by the kinase LKB1 and the scaffolding protein LIP1. J Biol Chem 286(1): 341-353.

Nakada, D., Saunders, T.L., and Morrison, S.J. (2010). Lkb1 regulates cell cycle and energy metabolism in haematopoietic stem cells. Nature 468, 653-658.

Nakau, M., Miyoshi, H., Seldin, M.F., Imamura, M., Oshima, M., and Taketo, M.M. (2002). Hepatocellular carcinoma caused by loss of heterozygosity in Lkb1 gene knockout mice. Cancer Res 62, 4549-4553.

O'Neill, G.M., Seo, S., Serebriiskii, I.G., Lessin, S.R., and Golemis, E.A. (2007). A new central scaffold for metastasis: parsing HEF1/ 
Cas-L/NEDD9. Cancer Res 67, 8975-8979.

Onozato, R., Kosaka, T., Achiwa, H., Kuwano, H., Takahashi, T., Yatabe, Y., and Mitsudomi, T. (2007). LKB1 gene mutations in Japanese lung cancer patients. Cancer Sci 98, 1747-1751.

Rodriguez-Nieto, S., and Sanchez-Cespedes, M. (2009). BRG1 and LKB1: tales of two tumor suppressor genes on chromosome 19p and lung cancer. Carcinogenesis 30, 547-554.

Roy, B.C., Kohno, T., Iwakawa, R., Moriguchi, T., Kiyono, T., Morishita, K., Sanchez-Cespedes, M., Akiyama, T., and Yokota, J. (2010). Involvement of LKB1 in epithelial-mesenchymal transition (EMT) of human lung cancer cells. Lung Cancer 70, 136-145.

Sanchez-Cespedes, M. (2007). A role for LKB1 gene in human cancer beyond the Peutz-Jeghers syndrome. Oncogene 26, 7825-7832.

Sanchez-Cespedes, M., Parrella, P., Esteller, M., Nomoto, S., Trink, B., Engles, J.M., Westra, W.H., Herman, J.G., and Sidransky, D. (2002). Inactivation of LKB1/STK11 is a common event in adenocarcinomas of the lung. Cancer Res 62, 3659-3662.

Shackelford, D.B., and Shaw, R.J. (2009). The LKB1-AMPK pathway: metabolism and growth control in tumour suppression. Nat Rev Cancer 9, 563-575.

Shaw, R.J., Kosmatka, M., Bardeesy, N., Hurley, R.L., Witters, L.A., DePinho, R.A., and Cantley, L.C. (2004). The tumor suppressor LKB1 kinase directly activates AMP-activated kinase and regulates apoptosis in response to energy stress. Proc Natl Acad Sci U S A 101, 3329-3335.

Sobottka, S.B., Haase, M., Fitze, G., Hahn, M., Schackert, H.K., and Schackert, G. (2000). Frequent loss of heterozygosity at the 19p13.3 locus without LKB1/STK11 mutations in human carcinoma metastases to the brain. J Neurooncol 49, 187-195.

Spano, J.P., Chouahnia, K., and Morere, J.F. (2004). Cyclooxygen- ase 2 inhibitors and lung carcinoma. Bull Cancer 91(Suppl 2), S109-S112.

Sun, Y., Ren, Y., Fang, Z., Li, C., Fang, R., Gao, B., Han, X., Tian, W., Pao, W., Chen, H., et al. (2010). Lung adenocarcinoma from East Asian never-smokers is a disease largely defined by targetable oncogenic mutant kinases. J Clin Oncol 28, 4616-4620.

Tomlinson, I.P., and Houlston, R.S. (1997). Peutz-Jeghers syndrome. J Med Genet 34, 1007-1011.

Upadhyay, S., Liu, C., Chatterjee, A., Hoque, M.O., Kim, M.S., Engles, J., Westra, W., Trink, B., Ratovitski, E., and Sidransky, D. (2006). LKB1/STK11 suppresses cyclooxygenase-2 induction and cellular invasion through PEA3 in lung cancer. Cancer Res 66, 7870-7879.

Weir, B.A., Woo, M.S., Getz, G., Perner, S., Ding, L., Beroukhim, R., Lin, W.M., Province, M.A., Kraja, A., Johnson, L.A., et al. (2007). Characterizing the cancer genome in lung adenocarcinoma. Nature 450, 893-898.

Wingo, S.N., Gallardo, T.D., Akbay, E.A., Liang, M.C., Contreras, C. M., Boren, T., Shimamura, T., Miller, D.S., Sharpless, N.E., Bardeesy, N., et al. (2009). Somatic LKB1 mutations promote cervical cancer progression. PLoS One 4, e5137.

Wu, Y., and Zhou, B.P. (2008). New insights of epithelialmesenchymal transition in cancer metastasis. Acta Biochim Biophys Sin (Shanghai) 40, 643-650.

Zeng, P.Y., and Berger, S.L. (2006). LKB1 is recruited to the p21/ WAF1 promoter by p53 to mediate transcriptional activation. Cancer Res 66, 10701-10708.

Zhang, S., Schafer-Hales, K., Khuri, F.R., Zhou, W., Vertino, P.M., and Marcus, A.I. (2008). The tumor suppressor LKB1 regulates lung cancer cell polarity by mediating cdc42 recruitment and activity. Cancer Res 68, 740-748. 